\title{
Psychological Stress Is a Risk Factor for Type 2 Diabetes Mellitus in College Students
}

\author{
Kukiat TUDPOR ${ }^{1}$, Chatcharida SAISAWAI, Sasina KAEWPHO and Amaraporn \\ JITSEEDA \\ Faculty of Public Health, Mahasarakham University, Maha Sarakham, Thailand
}

\begin{abstract}
Type 2 diabetes mellitus (T2DM) is multifactorial disease. This crosssectional study was aimed to investigate relationship between stress and risk for T2DM in college students. Seven-hundred participants (350 T2DM risk and 350 non-T2DM risk groups). Stress index levels and heart rate variability (HRV) were respectively measured as primary and secondary outcomes. Results showed that both T2DM-risk and non-T2DM-risk groups had temporary stress, but the T2DMrisk group had significantly higher level of psychological stress $(\mathrm{P}<.001)$. For the HRV, the T2DM-risk group had significantly lower levels of parasympathetic proxies (lnHF, SDNN, and RMSSD) $(\mathrm{P}<.001)$. Chi-square $\left(\chi^{2}\right)$ test showed significant correlation of the stressful state with T2DM risk $\left(\chi^{2}=159.372, \mathrm{P}<.001\right.$, odds ratio $(\mathrm{OR})=9.326)$. In conclusion, psychological stress is a risk factor for T2DM in college students. Early detection, monitoring, and treatments of psychological stress should be implemented in this group of population.
\end{abstract}

Keywords Psychological stress, Type 2 diabetes mellitus, Young adulthood

\section{Introduction}

Type 2 diabetes mellitus (T2DM) is major non-communicable disease worldwide [2]. According to Clinical Practice Guideline (CPG) for Diabetes 2017 of the Diabetes Association of Thailand, risk score checklist for T2DM in Thai people has been developed based on advancing age, masculinity, family history, body mass index (BMI), central obesity, and hypertension [1]. Young adults (18-26 years old) are in a critical period in life as they have to think about their future career paths and economic security [12]. Hence, they are prone to depression, and psychological stress [8]. However, their needs during this transitional period were not concerned. Psychological stress might cause T2DM via stress hormone-induced insulin insensitivity [7]. This study investigated relationship between psychological stress and T2DM risk in young adult individuals by using a novel non-invasive technology device for stress index measurement on a fingertip.

${ }^{1}$ Corresponding Author: Kukiat Tudpor, Faculty of Public Health, Mahasarakham University, Kham Rieng, Kantarawichai, Maha Sarakham, Thailand 44150; E-mail: kukiat.t@msu.ac.th 


\section{Methods}

\subsection{Study Design and Participants}

Seven hundred college students from Mahasarakham University were recruited into this cross-sectional study. Inclusion criteria were being bachelor students and signed consent form. All procedures were approved by 2 reviewers of the Ethical Review Committee for Human Research, Mahasarakham University, and endorsed by the chairperson Dr.Ratree Sawangjit (No.276/2563). Exclusion criteria were psychiatric disease, metabolic disease, and recent major trauma. T2DM risk score was obtained from a checklist from the CPG [4]. The risk score was ranged from $0-17$. The participants with score $<6$ and $\geq 6$ were divided into non-T2DM-risk and T2DM-risk groups (Table 1).

Table 1 Risk factors for T2DM and risk score

\begin{tabular}{lll}
\hline \multicolumn{1}{c}{ Risk factors for T2DM } & Risk score \\
\hline Age (years old) & $34-39$ & 0 \\
& $40-44$ & 0 \\
& $45-49$ & 1 \\
Sex & $\geq 50$ & 2 \\
& Female & 0 \\
Body mass index & Male & 2 \\
& $<23 \mathrm{~kg} / \mathrm{m}^{2}$ & 0 \\
Waist circumference & $23-27.4 \mathrm{~kg} / \mathrm{m}^{2}$ & 3 \\
& $\geq 27.5 \mathrm{~kg} / \mathrm{m}^{2}$ & 5 \\
Hypertension & Male $<90 \mathrm{~cm}$, Female $<80 \mathrm{~cm}$ & 0 \\
& Male $\geq 90 \mathrm{~cm}$, Female $\geq 80 \mathrm{~cm}$ & 2 \\
Type 2 diabetes mellitus in first-degree relative & No & 0 \\
& Yes & 2 \\
\end{tabular}

\subsection{Outcome Measurements}
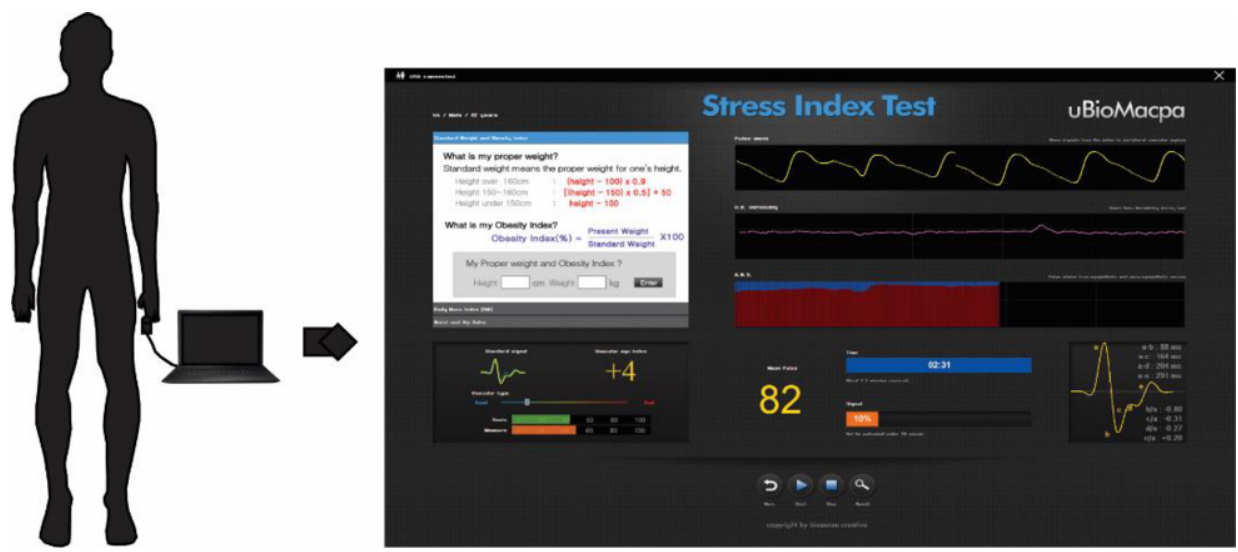

Figure 1 Non-invasive technology device for stress index measurement on a fingertip

Primary outcome (stress index) and secondary outcomes (HRV) were measured by photo-plethysmography (PPG) on the participant's left index finger tips connected to the uBioMacpa software as previously detailed [11] (Figure 1). The stress index scores were classified as 5 groups: no stress $(<25)$, temporary stress $(25-34)$, primary stress $(35-44)$, 
accumulative stress (45-59), and chronic stress $(\geq 60)$. The HRV parameters include sympathetic proxies [low frequency ( $\operatorname{lnLF})$ and low/high frequency ratio (LF/HF ratio)] and parasympathetic proxies [high frequency ( $\operatorname{lnHF})$, standard deviation of all normal R$\mathrm{R}$ intervals (SDNN), and square root of the mean of the squared successive differences in R-R intervals (RMSSD)]. Statistical analyses were independent t-test and $\chi^{2}$ test.

\section{Results}

\subsection{T2DM-risk group had higher level of psychological stress}

Both T2DM-risk and non-T2DM-risk groups had temporary stress, but T2DM-risk group had significantly higher level of psychological stress $(\mathrm{P}<.001)$. When further subdivided into the stressful subgroups (stress index $\geq 25$ ), the T2DM-risk group had primary stress whilst the non-T2DM-risk group had temporary stress. Moreover, the T2DM-risk group also had statistically faster pulse rate. For the HRV, the T2DM-risk group had significantly lower lnHF, SDNN, and RMSSD $(\mathrm{P}<.001)$ (Table 2).

Table 2 Stress index and heart rate variability parameters in non-risk-T2DM and risk-T2DM

\begin{tabular}{lccc}
\hline \multicolumn{1}{c}{ Parameter } & $\begin{array}{c}\text { Non-T2DM risk } \\
(\mathbf{n}=\mathbf{3 5 0})\end{array}$ & $\begin{array}{c}\text { T2DM risk } \\
(\mathbf{n}=\mathbf{3 5 0})\end{array}$ & P-value \\
\hline Stress index & $25.20 \pm 5.06$ & $33.96 \pm 8.06$ & $<.001$ \\
$\quad$ Non-stressful & $21.93 \pm 2.37$ & $22.37 \pm 2.41$ & .25 \\
$\quad$ Stressful & $30.00 \pm 4.03$ & $35.79 \pm 7.03$ & $<.001$ \\
Pulse rate (beat/min) & $76.59 \pm 7.47$ & $80.43 \pm 10.99$ & $<.001$ \\
$\ln$ LF (ms $\left.{ }^{2}\right)$ & $7.97 \pm 0.61$ & $8.57 \pm 7.88$ & .16 \\
$\ln H F\left(\mathrm{~ms}^{2}\right)$ & $7.20 \pm 0.48$ & $6.93 \pm 0.64$ & $<.001$ \\
LF/HF ratio & $1.11 \pm 0.08$ & $1.12 \pm 0.09$ & .17 \\
SDNN (ms) & $65.74 \pm 17.15$ & $54.60 \pm 23.34$ & $<.001$ \\
RMSSD (ms) & $51.25 \pm 16.42$ & $42.52 \pm 23.09$ & $<.001$ \\
\hline
\end{tabular}

\subsection{Stressful State was Correlated T2DM Risk}

Further analysis revealed that $\chi^{2}$ test revealed that a stressful state was significantly correlated T2DM risk $\left(\chi^{2}=159.37, \mathrm{P}<.001, \mathrm{OR}=9.32\right)$ (Table 3$)$.

Table 3 Association between stress index and risk for T2DM

\begin{tabular}{lcccccc}
\hline Stress index & $\begin{array}{c}\text { T2DM } \\
\text { risk (\%) }\end{array}$ & $\begin{array}{c}\text { Non-T2DM } \\
\text { risk (\%) }\end{array}$ & Total & $\chi^{\mathbf{2}}$ & P-value & OR (95\% CI) \\
\hline Non-stressful & 141 & $302(86.29)$ & 443 & 159.37 & $<0.001$ & $9.32(6.42-13.52)$ \\
& $\left(\begin{array}{c}40.29) \\
209\end{array}\right.$ & $48(13.71)$ & 257 & & & \\
Stressful & $\left(\begin{array}{c}59.71) \\
350\end{array}\right.$ & 350 & 700 & & & \\
Total & & & & & \\
\hline
\end{tabular}




\section{Discussion and Conclusion}

Risk for Type 2 Diabetes Mellitus in Transitional Age Youth (TAY): T2DM is caused by both genetic and non-genetic factors. The non-genetic factors consist of modifiable (physical activity, nutrition, smoking, alcohol drinking etc.) and non-modifiable factors (race, age, and sex etc.) [3]. Psychological stress is a modifiable factor causing T2DM in adults and elderly persons [5]. This present study was first to show a high psychological stress level in college students with high-risk score of T2DM. Age of the participants in this study (19-22 years old) is considered as TAY. An MRI study shows that prefrontal cortex (part of the brain for decision making) in TAY is still developing, implying stressful challenges of making decision in college students [6].

Possible Mechanisms of Psychological Stress-Induced Insulin Resistance in TAY: Psychological stress can be measured by psychological marker (stress questionnaire), biochemical marker (plasma cortisol), or physiological marker (HRV) [9]. Here we found that parasympathetic proxies of the HRV in the T2DM-risk group were lower than the non-T2DM-risk group. An increase in stress index in the T2DM-risk group might be due to reduced parasympathetic tone and increased sympathetic tone as shown by rapid pulse rate. Lastly, the participants with high stress index had 9 times greater likelihood of T2DM risk. Mechanisms of T2DM risk might be explained by psychological stressinduced insulin resistance via phosphorylated protein kinase B downregulation [10].

Conclusion: To conclude, psychological stress is a risk factor for T2DM in college students. Therefore, early detection, monitoring, and treatments of psychological stress should be implemented in this group of population.

\section{References}

[1] Aekplakorn W, Bunnag P, Woodward M, Sritara P, Cheepudomwit S, etal. A risk score for predicting incident diabetes in the Thai population. Diabetes Care 2006; 29: 1872-1877.

[2] Apidechkul T. Prevalence and factors associated with type 2 diabetes mellitus and hypertension among the hill tribe elderly populations in northern Thailand. BMC Public Health 2018; 18: 694.

[3] Deshpande AD, Harris-Hayes M, Schootman M. Epidemiology of diabetes and diabetes-related complications. Phys Ther 2008; 88: 1254-1264.

[4] Diabetes Association of Thailand. Clinical Practice Guidelines for Diabetes Diabetes Association of Thailand, 2017.

[5] Diez Roux AV, Jacobs DR, Kiefe CI, Coronary S. Neighborhood characteristics and components of the insulin resistance syndrome in young adults - The coronary artery risk development in young adults (CARDIA) study. Diabetes Care 2002; 25: 1976-1982.

[6] Giedd JN. The teen brain: insights from neuroimaging. J Adolesc Health 2008; 42 (4): 335-343.

[7] Hackett RA, Steptoe A. Type 2 diabetes mellitus and psychological stress - a modifiable risk factor. Nat Rev Endocrinol 2017; 13(9): 547-560.

[8] Hafner H, Maurer K, Loffler W, Riecher-Rossler A. The influence of age and sex on the onset and early course of schizophrenia. Br J Psychiatry 1993; 162: 80-86.

[9] Kim HG, Cheon EJ, Bai DS, Lee YH, Koo BH. Stress and Heart Rate Variability: A Meta-Analysis and Review of the Literature. Psychiatry Investig. 2018; 15(3): 235-245.

[10] Li L, Li X, Zhou W, Messina JL. Acute psychological stress results in the rapid development of insulin resistance. J Endocrinol. 2013; 217(2): 175-184.

[11] Shin Y, Ham J, Cho H. Experimental Study of Thermal Comfort Based on Driver Physiological Signals in Cooling Mode under Summer Conditions. Applied Sciences 2021; 11(2): 845.

[12] Wilens TE, Rosenbaum JF. Transitional aged youth: a new frontier in child and adolescent psychiatry. J Am Acad Child Adolesc Psychiatry 2013; 52(9), 887-890. 